\title{
Supplementation With Galactooligosaccharides and Inulin Increases Bacterial Translocation in Artificially Reared Newborn Rats
}

\author{
EMMANUEL BARRAT, CATHERINE MICHEL, GUILLAUME POUPEAU, AGNES DAVID-SOCHARD, MARTINE RIVAL, \\ ANTHONY PAGNIEZ, MARTINE CHAMP, AND DOMINIQUE DARMAUN
}

UMR 1280, Physiologie des Adaptations Nutritionnelles [E.B., C.M., G.P., A.D.-S., M.R., A.P., M.C., D.D.], Université de Nantes, Nantes, F-44093; CRNH [E.B., C.M., D.D.], Nantes, F-44093; IMAD [C.M., M.C., D.D.], CHU de Nantes, Nantes, F-44093, France

\begin{abstract}
Supplementation of formulas with prebiotics enhances the growth of lactate producing bacteria, and fecal lactate, and acetate levels in infants. High concentrations of organic acids in intestinal lumen have, however, been shown to impair the intestinal barrier function. To determine whether stimulating the colonic microbiotal metabolism with prebiotics would impair the neonatal intestinal barrier function, artificially reared rats were fed milk formula with or without a mixture of galactooligosaccharides/inulin (GOS/Inulin, $88 / 12 ; 5.6 \mathrm{~g} / \mathrm{L}$ ) from the 7 th d of life (d7) until weaning (d20). At $\mathrm{d} 18$, GOS/inulin supplementation had increased the concentrations of acetate and lactate in colonic lumen. Although neither ileumassociated microbiota nor colonic permeability (assessed in Ussing chambers), nor the expression of tight junction claudin- 2 and claudin-3 mRNA were altered, GOS/inulin supplementation was associated with increased bacterial translocation (BT) toward spleen. None of these effects persisted at $\mathrm{d} 40$. We conclude that GOS/inulin supplementation may increase BT in an immature gut. The balance between the potential infectious risk of BT vs. its putative beneficial effect on the maturation of neonatal immune system clearly warrants further study. (Pediatr Res 64: 34-39, 2008)
\end{abstract}

A $\mathrm{s}$ breast-feeding is associated with multiple health benefits, attempts have been made to induce alterations in intestinal microbiota of formula-fed infants designed to mimic that observed in breast-fed infants. This has been part of the rationale for supplementing some infant feeding formulas with prebiotics, mainly a mixture of galactooligosaccharides (GOS) and inulin. This GOS/inulin mixture indeed has been shown to have a prebiotic effect by increasing the proportion of bifidobacteria and lactobacilli in feces [reviewed in (1)], and to enhance the production of acetate and lactate in the colon of formula-fed infants, to levels similar to those observed in breast-fed babies (2). Recent studies found GOS/inulin may confer protection against infections (3), and decrease the incidence of atopic dermatitis for babies at risk (4) but the true benefits of GOS/inulin remain to be clearly established.

As immaturity of the intestinal barrier function is thought to contribute to the pathogenesis of necrotizing enterocolitis (5), it is of interest to reinforce the intestinal barrier function of

Received November 27, 2007; accepted February 11, 2008.

Correspondence: Martine Champ, Ph.D., CHU- Hôtel Dieu, HNB1- UMR PhAN, Physiologie des Adaptations Nutritionnelles, Place Alexis Ricordeau, 44093 Nantes Cedex 1, France; e-mail: martine.champ@univ-nantes.fr

This work was supported in part, by doctoral fellowship grants from: INRA and Région Pays de la Loire, France, from Nutricia - Société Française de Nutrition Entérale et Parentérale, and from the Institut Benjamin Delessert (E.B.). newborns. Yet, the effect of prebiotic supplementation on barrier function has largely remained unexplored, with the exception of a nonrandomized study devoid of control group (CTL) which reported no impact (6).

Whether prebiotics will impact positively or negatively on the intestinal barrier function of infants is not predictable from the literature due to conflicting results.

On the one hand, prebiotics are fermented into short chain fatty acids (SCFA), particularly butyrate, which plays a key role in the maintenance of mucosal homeostasis in adult colon (7), and reduces intestinal permeability and bacterial translocation (BT) in adult rats with experimental colitis (8).

On the other hand, butyrate production appears deleterious in infants since 1) an over-production of butyrate has been associated with the onset of necrotizing enterocolitis (9); and 2) colonic perfusion of high concentrations of butyrate (300 $\mathrm{mM}$ ) induced mucosal damage in pups younger than $20 \mathrm{~d}$ of age $(10,11)$. Such an impact could be considered as irrelevant since neonatal gut microbiota does not produce butyrate (12), and therefore prebiotic supplementation solely enhances lactate and acetate production in infants (2). Colonic perfusions of highly concentrated $(300 \mathrm{mM})$ acetate, however, also causes mucosal damage in rat pups $(10,11)$ and elevation of organic acid levels have been linked to the increased incidence of Salmonella translocation observed in adult rats fed a lowcalcium diet supplemented with prebiotics $(13,14)$.

We therefore speculated that the increased production of lactate and acetate induced by a supplementation with GOS/ inulin could impair the intestinal barrier function of newborns. Because of the limitations intrinsic to the study of intestinal barrier function in human infants, we chose to use the "pup in the cup" model (15). Newborn rats were artificially reared and fed a formula with or without a mixture of GOS/inulin from postnatal day 7 (d7) until weaning (d20). We observed an increased incidence of BT in rats fed GOS/inulin at d18 and then evaluated the persistence of this effect (at d40) after weaning rats with a GOS/inulin-free diet.

\footnotetext{
Abbreviations: BT, bacterial translocation; CFUs, colony forming units; CTL, control group; GOS, galactooligosaccharides; GOS/In, GOS/inulin fed group; SCFA, short chain fatty acids
} 


\section{METHODS}

Animals. Experiments were carried out in accordance with the recommendations of the local Animal Care and Use Committee of Nantes (France). The animal facilities were approved by the government agency in charge of experimental facilities at the French Department of Agriculture, the investigators were accredited by the National Veterinary Agency, and the "pup in the cup" model was approved as well by a veterinary practitioner from the National School of Veterinary Medicine. Male newborn rats, originated from 30 pregnant time-dated Sprague-Dawley dams (Elevage Janvier, Le Genest St Isle, France), were used in three consecutive sets of experiments. Ninety-five male pups ( $n=30 ; n=30$; and $n=35$ in sets no. 1,2 , and 3, respectively) underwent noninvasive gastrostomy $5 \mathrm{~d}$ after delivery (d5) as described earlier (16). Four times a day, urination and defecation were induced by gentle stimulation of the anal/genital area of each rat pup. The amount of feces excreted was evaluated semiquantitatively according to a $0-3$ scale from $\mathrm{d} 7$ until $\mathrm{d} 16$, at which time the rats were able to defecate alone. During the third set of experiments, seven male pups from the same litters as gastrostomized pups and raised by their mother were also included for comparison.

Milk formulas preparation and feeding. The milk formulas were prepared from powdered rat milk replacer (Wombaroo Food Products, Glen Osmond, Australia). A total of $10.9 \mathrm{~g} / \mathrm{L}$ of Vivinal GOS (Friesland Foods Domo, Zwolle, The Netherlands) i.e., $4.9 \mathrm{~g} / \mathrm{L}$ of pure GOS and $0.75 \mathrm{~g} / \mathrm{L}$ of Beneo HP (Orafti, France) i.e., $0.70 \mathrm{~g} / \mathrm{L}$ of pure inulin were added to the supplemented milk formula. Because Vivinal GOS contains up to 23 and $22 \%$ of lactose and glucose, respectively, the control milk formula was supplemented with 2.5 $\mathrm{g} / \mathrm{L}$ of lactose and $2.4 \mathrm{~g} / \mathrm{L}$ of glucose. The osmolarities of the two formulas were comparable (362 vs. $375 \mathrm{mOsml} / \mathrm{L}$; CTL vs. GOS/inulin). During the first postoperative day, the rats were fed the half strength control milk formula. On d6, all rats were fed control milk formula. At d7, the rats were randomly assigned either to the CTL receiving the control milk formula, or to the supplemented group (GOS/inulin) receiving the GOS/inulin supplemented milk formula. Every morning, the daily amount of milk formula to be infused was determined according to the age of the rats (from $3.6 \mathrm{~mL}$ at d5 to $15.1 \mathrm{~mL}$ at d20). At d20, the rats were weaned to solid food (D03; Safe, Augy, France) and tap water. Finally, because of pups mortality or exclusion (see the Result section), and considering the three sets of experiments in aggregate, a total of 20 and 22 pups included in the CTL and GOS/inulin, respectively, were anesthetized with isoflurane at $\mathrm{d} 18$, and killed by decapitation; 14 and 14 rats from CTL and GOS/inulin were also killed at $\mathrm{d} 40$ by $\mathrm{CO}_{2}$ inhalation. During the third set of experiments, the seven rats raised by their mothers were also killed at d18.

Bacterial translocation and ileum-associated microbiota. Immediately after killing (d18: $n=20 \mathrm{CTL}, n=22 \mathrm{GOS} /$ inulin and $n=7$ mother-reared rats; d40: $n=14 \mathrm{CTL}$ and GOS/inulin), the spleen was excised using sterile procedure, weighed, and ground in sterile Ringer with a pellet pistol. Five centimeter of distal ileum were also excised (d18: $n=8$ CTL, $n=10$ GOS/inulin and $n=7$ mother-reared rats), weighed, rinsed consecutively in three sterile saline baths and ground in sterile Ringer with a pellet pistol. Hundred microliters of the homogenate and of 10 -fold serial dilutions in Ringer were plated onto four separate plates (Biokar diagnostic, Beauvais, France) that included Nutrient Agar and MacConkey media incubated in aerobic conditions, Wilkins and Chalgren and MRS agar incubated in anaerobic conditions $\left(\mathrm{N}_{2} / \mathrm{CO}_{2} / \mathrm{H}_{2}: 85 / 10 / 5\right)$. After 24 to $96 \mathrm{~h}$ of incubation, the number of colony-forming units (CFUs) per plate was counted. The total number of CFUs per spleen (BT intensity) or per gram of ileum was calculated. Growth in spleen was considered positive if greater than 30 CFUs/spleen were identified. BT incidence was defined as the percentage of rats that presented a positive bacterial culture in spleen.

Colonic permeability in Ussing chambers. Immediately after killing, cecum, proximal, and distal colons (d18: $n=18, \mathrm{~d} 40: n=14$ ) were weighed, removed, and opened along the mesenteric border into a flat sheet. Tissues were rinsed in modified Krebs-Henseleit buffer (Sigma-Aldrich Chimie Sarl, St Quentin Fallavier, France), and mounted in Ussing chambers (Physiologic Instruments, San Diego, CA) less than 20 min after killing, exposing $0.25 \mathrm{~cm}^{2}$ to $1 \mathrm{~mL}$ of circulating oxygenated Krebs buffer at $38^{\circ} \mathrm{C}$. After $20 \mathrm{~min}$, FITC-labeled 4-KDa dextran (Sigma-Aldrich Chimie Sarl, St Quentin Fallavier, France) was added at the mucosal side $(4.4 \mathrm{mg} / \mathrm{mL}$ final concentration) and fluorescence was measured at the serosal side after 120 min. Fluxes of FITC-Dextran were expressed as $\mu \mathrm{g} / \mathrm{h} / \mathrm{cm}^{2}$. The surface areas $(n=12$ per group) of the entire colon and that of the entire cecum were measured as the surfaces of a trapezoid of a quadrilateral, respectively.

Luminal organic acids concentration in the colon. At d18 $(n=20 \mathrm{CTL}$, $n=22 \mathrm{GOS} / \mathrm{inulin}$ ), the luminal contents from the cecum and the colon were pooled together; at $\mathrm{d} 40$ ( $n=14$ per group), the cecal content alone was analyzed. Short-chain fatty acids (SCFA) analysis was performed by capillary gas chromatography [adapted from Ref. (17)] on supernatant of samples diluted in oxalic acid and centrifuged at $8000 \mathrm{~g}$ for $10 \mathrm{~min}$. D-lactate, L-lactate
Table 1. Sequences and efficiencies of forward $(F)$ and reverse $(R)$ primers used for real-time PCR

PCR

\begin{tabular}{ccc}
\hline Gene & Sequences of primers $\left(5^{\prime}-3^{\prime}\right)$ & $\begin{array}{c}\text { PCR } \\
\text { efficiency }(\%)\end{array}$ \\
\hline ZO-1 (Tjp-1) & F: GGA AAC CGC AAA & 80 \\
& CTG ATG CTA TGG & \\
& R: AAC TGG CTG GCT & \\
GTA CTG TGAG & 100 \\
Claudin-2 & F: TCC TCT GCT TTT & \\
& CCT GCT CAC C & \\
R: CAC TCT TGG CTT & 100 \\
Tlaudin-3 & TGG GCT GTT G & \\
& F: ACA CCG CAC CAT & \\
& CAC CAC TAC & \\
R: TCT TCC AGC CTA & \\
GCA AGC AGA C & \\
FAPDH & CAG TAT GAC TCT & \\
& ACC CAC GGC A & \\
& R: ATC TCG CTC CTG & \\
GAA GAT GGT G &
\end{tabular}

Primers were designed with Beacon Designer software (Premier Biosoft International). Specificity of each couple of primers was tested using BLAST software (NCBI).

concentrations were determined using an assay kit according to the manufacturer's instructions (Biosentec, Toulouse, France). Results were expressed as $\mu \mathrm{mol}$ of organic acid/g of luminal content.

Reverse-transcription PCR. Samples (10-20 mg) of distal colon (d18: $n=12$ per group) were rinsed in sterile saline, snap frozen in liquid nitrogen, and stored at $-70^{\circ} \mathrm{C}$ until analysis. Tissue RNA was extracted with the NucleoSpin RNA kit (Macheray-Nagel EURL, Hoerdt, France) following the manufacturer's instructions. cDNA was synthesized from $2 \mu \mathrm{g}$ of total RNA in $25 \mu \mathrm{L}$ final volume using random primers and MMLV reverse transcriptase (Promega, Madison, WI) according to the manufacturer's instructions. Realtime PCR was performed in the presence of $150 \mathrm{ng}$ of cDNA in final volume of $15 \mu \mathrm{L}$ containing $250 \mathrm{nmol} / \mathrm{L}$ of each specific primer using QuantiTect SYBR Green PCR Kit (Qiagen, Courtaboeuf, France). Amplification (45 cycles: $30 \mathrm{~s}$ at $95^{\circ} \mathrm{C}$ and $10 \mathrm{~s}$ at $60^{\circ} \mathrm{C}$ ) was performed with the iCycler iQ real-time PCR detection system instrument (Bio-Rad Laboratories, Hercules, CA). Sequences and PCR efficiency of primers are stated in Table 1. Results were expressed relative to GAPDH gene expression compared with the CTL, according to the $2^{-\Delta \Delta \mathrm{Ct}}$ (where $\mathrm{Ct}$ is the cycle threshold) calculation method, taking into account the efficiency of the primers if not $100 \%$.

Histomorphometry. Shortly after killing, cecal and distal colonic mucosa (d18: $n=12$ per group) opened longitudinally were rinsed with sterile $0.9 \%$ saline, and rolled around a wooden stick as described by the 'Swiss roll' technique (18). Sections $(5 \mu \mathrm{m})$ of formaldehyde-fixed paraffin-embedded tissues were stained with hematoxylin and eosin, and observed by light microscopy. For each tissue, the length and height of 10 crypts at two different spots and the thickness of the nonepithelial layer (sub-mucosa + muscularis + serosa) at 10 spots were recorded. The mean values of the 10 measurements for each rat were used for statistical analysis.

Statistical analysis. Values are given as medians (or absolute values for $\mathrm{BT}$ incidence) and the interquartile ranges (Q1-Q3) are parenthesized. Statistics were done using Statview v.2. The effect of GOS/inulin supplementation was analyzed with the Mann-Whitney test. During the third set of experiments, differences between mother reared rats and the two groups of artificially reared pups were first analyzed with the Kruskal-Wallis test, then by pair with Mann-Whitney test. Statistical analysis of BT was performed with the $\chi^{2}$ test. Differences were considered statistically significant when $p$-value $<0.05$.

\section{RESULTS}

Rat pup growth. Fifteen of the 95 rats had to be excluded from the experiments since they pulled out their cannula, and three rats from the GOS/inulin group (GOS/In) died at 11,14 , and $17 \mathrm{~d}$ of life without any obvious reason, and while their cannulas were in place. $\chi^{2}$ Analysis did not 
reveal any statistical difference in mortality between the two groups ( $p=0.08)$.

As the same pump was used to deliver both formulas, milk intake was similar between GOS/inulin and CTL. Weight gain between d5 and d18 [19.1 (3.1) g vs. 18.5 (3.6) g], body weight [34.7 (2.7) g vs. 33.5 (3.9) g], and body length [17.3 $(1.0) \mathrm{cm}$ vs. $17.0(0.5) \mathrm{cm}]$ at d18 were similar in CTL and GOS/inulin.

Weights of the brain, liver, spleen, pancreas, lungs, and femur did not differ between the two experimental regimens (data not shown). A small, although not significant, increase $(6 \%)$ in length of the entire small intestine $(p=0.09)$ and of the entire colon $(p=0.12)$ was observed in GOS/inulin, compared with CTL (data not shown).

Trophic effect of GOS/inulin on large intestine. At d18, the surface area and weight of ceca were 1.7 and 1.5 times greater ( $p=0.02$ and $p=0.0001$, respectively) in GOS/inulin than in CTL (Fig. $1 A$ and $B$ ) but the weight/surface ratio was not different. The weight and surface area of the colon did not differ between the two experimental regimens. The increased weight and surface of the cecum of $18 \mathrm{~d}$-old rats did not last through d40 (weight: 1.3 (0.8) vs. 1.4 (0.5) g; surface: 8.5 (1.6) vs. 9.0 (2.5) $\mathrm{cm}^{2}$; for CTL and GOS/inulin, respectively).

The height and width of cecal and colonic crypts, as well as the thickness of the nonepithelial layer did not differ among the two groups at d18 (data not shown).
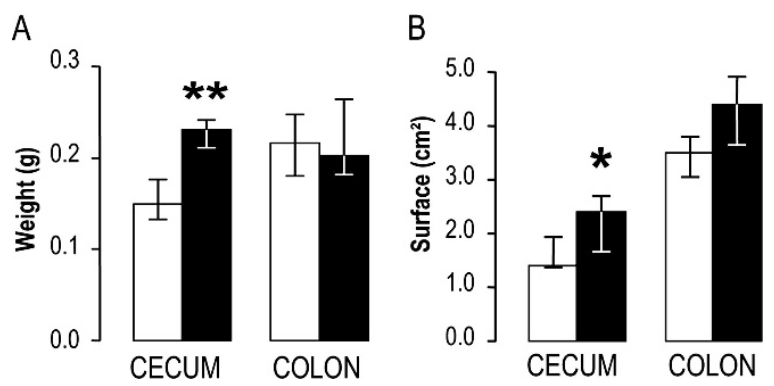

Figure 1. (A) Weight of cecum and colon at d18 ( $n=12$ per group); $(B)$ Surface area of the cecum and colon at $\mathrm{d} 18$ ( $n=12$ per group). Rats were artificially fed rat milk substitute with (ם) or without $(\square)$ GOS/inulin from d7 to d18. Bars represent medians and error bars the first and third quartiles. Different $v s$. CTL (Mann-Whitney): * $p<0.05$; ** $p<0.01$.
Effect of GOS/inulin on colonic microbiota metabolism. Stool emission (number and volume of stools per day) was increased in GOS/inulin, compared with CTL $(p<0.05)$, from $\mathrm{d} 9$ to $\mathrm{d} 15$ (Table 2), and feces of GOS/inulin were noted to be softer than those from CTL (results not shown). The total amount of luminal contents in the cecum and colon from GOS/inulin was also 1.6 times heavier, compared with CTL, at d18 (Table $2 ; p=0.02$ ).

The concentrations of total lactate and of total SCFA were 1.6 and 1.2 times higher $(p=0.009$ and $p=0.01)$, respectively, in GOS/inulin compared with CTL. Only L-lactate concentration was significantly increased in GOS/inulin compared with CTL (Table 2). Median D-lactate concentrations were below the limit of quantification of the assay. Among SCFA, only acetate (representing $90 \%$ of total SCFA in both groups) concentration was significantly increased (Table 2). The enhanced production of acetate observed in the GOS/ inulin, compared with CTL, did not last through d40.

Effect of GOS/inulin on intestinal barrier function. Considering all three experiments in aggregate at d18, incidence of BT was higher in GOS/inulin compared with CTL, both for aerobes and anaerobes (Fig. $2 A ; p=0.02$ and $p=0.005$, respectively). However, the number of aerobes and anaerobes found in the spleen (i.e., BT intensity) did not differ between CTL and GOS/In (2.0 vs. $2.2 \log _{10}$ and 2.0 vs. $2.4 \log _{10}$ CFUs; respectively). BT incidence and intensity was similar in CTL and GOS/inulin for lactic acid producing bacteria and enterobacteria (Fig. 2A). The increase in BT incidence observed in GOS/inulin was not observed anymore at d40 (Fig. 2A).

Data from the sole third set of experiments (where motherreared pups were included) were consistent with these trends at d18 (Table 3) but also emphasized that BT was specifically increased by GOS/inulin supplementation. Indeed, BT was not increased in CTL artificially reared pups compared with mother reared pups $(p=0.60)$ but was increased in GOS/ inulin compared with both CTL and mother-reared pups ( $p=$ 0.02 and $p=0.03$ respectively, Table 3 ).

The paracellular permeability to macromolecules (flux of 4 KDa Dextran measured in Ussing chambers) did not differ between GOS/inulin and CTL in cecum, proximal or distal colon at d18 and d40 (Fig. 2B). Besides, at d18, the colonic expression of the genes encoding for tight junction proteins

Table 2. Effect of dietary GOS/inulin supplementation on stool emission, weight of ceco-colonic luminal contents, and organic acids concentrations in ceco-colonic luminal content at $d 18$ and $d 40$

\begin{tabular}{|c|c|c|c|c|}
\hline & \multicolumn{2}{|c|}{ d 18} & \multicolumn{2}{|c|}{ d 40} \\
\hline & CTL & GOS/In & CTL & GOS/In \\
\hline Stool emission between $\mathrm{d} 9-\mathrm{d} 15$ & + & $++*$ & n.d. $\dagger$ & n.d. \\
\hline Ceco-colonic luminal content $\$$ (g) & $0.08(0.07)$ & $0.13(0.07)^{*}$ & $3.77(0.77)$ & $3.32(0.67)$ \\
\hline D-Lactate $\ddagger\left(\mu \mathrm{mol} \mathrm{g}^{-1}\right)$ & $<3.7$ & $<3.7$ & n.d. & n.d. \\
\hline L-Lactate $\$(\mu \mathrm{mol} \mathrm{g}-1)$ & $4.3(1.1)$ & $6.4(2.7) \S$ & n.d. & n.d. \\
\hline Acetate $\ddagger\left(\mu \mathrm{mol} \mathrm{g}^{-1}\right)$ & $57(24)$ & $69(18)^{*}$ & $70(3)$ & $66(2)$ \\
\hline Propionate $\$\left(\mu \mathrm{mol} \mathrm{g}{ }^{-1}\right)$ & $2(1)$ & $2(1)$ & $13(3)$ & $13(5)$ \\
\hline Butyrate $\neq\left(\mu \mathrm{mol} \mathrm{g}^{-1}\right)$ & $3(1)$ & $4(3)$ & $33(8)$ & $35(14)$ \\
\hline Other SCFA $\ddagger\left(\mu \mathrm{mol} \mathrm{g}^{-1}\right)$ & $1(2)$ & $1(2)$ & $1(1)$ & $1(2)$ \\
\hline
\end{tabular}

\footnotetext{
* Different $v s$. CTL $(p<0.05)$.

$\dagger$ n.d., not determined.

$\$$ Results are shown as medians; interquartiles (Q1-Q3) are parenthesized.

$\S$ Different vs. CTL $(p<0.01)$.
} 


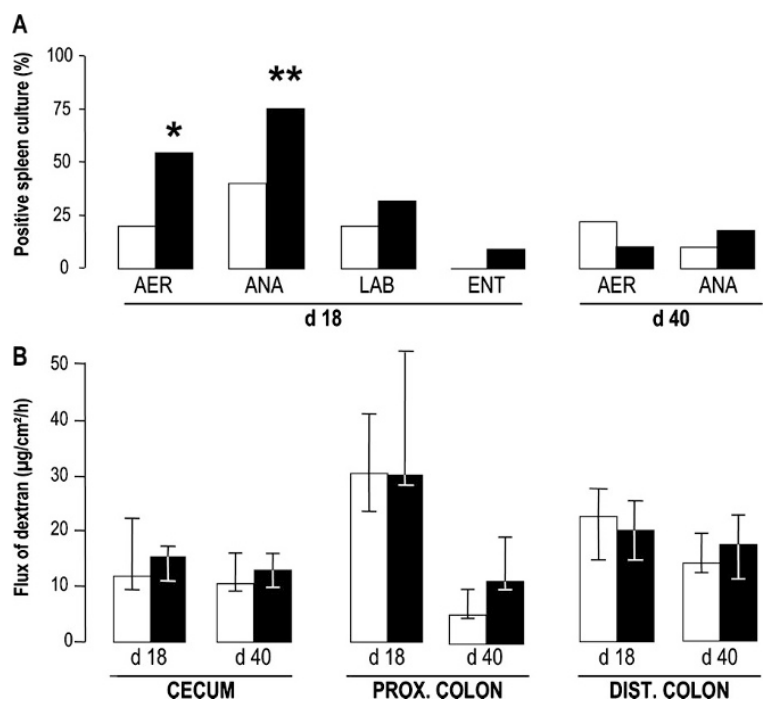

Figure 2. (A) Translocation of aerobes (AER), anaerobes (ANA), lactic acid producing bacteria (LAB), and enterobacteria (ENT) toward spleen at d18 (minimum $n=20$ per group) and d40 ( $n=14$ per group). (B) Paracellular permeability of cecum, proximal, and distal colon to $4 \mathrm{KDa}$ FITC-Dextran measured in Ussing chambers at $\mathrm{d} 18(n=18$ per group $)$ and $\mathrm{d} 40(n=14 \mathrm{per}$ group). Rats were artificially fed rat milk substitute with ( $\square$ ) or without ( $\square$ ) GOS/inulin from $\mathrm{d} 7$ to $\mathrm{d} 20$, then were weaned with normal GOS/inulin-free diet at $\mathrm{d} 20$. Bars represent absolute values $(A)$ or medians $(B)$ and error bars the first and third quartiles. Different $v s$. CTL $\left(\chi^{2}\right): * p<0.05 ; * * p<0.01$.

Table 3. Bacterial translocation and ileal mucosa-associated bacteria in mother-reared rats, CTL and GOS/In during the third set of experiments at $d 18$

\begin{tabular}{llll}
\hline & Mother reared & CTL & GOS/In \\
\hline $\begin{array}{l}\text { Bacterial translocation, } \\
\text { positive spleen cultures } \ddagger\end{array}$ & & & \\
$\quad$ Aerobes & $3 / 7(43 \%)$ & $3 / 8(38 \%)$ & $6 / 10(60 \%)$ \\
Anaerobes & $2 / 7(29 \%)$ & $2 / 8(25 \%)$ & $8 / 10(80 \%)^{* \dagger}$ \\
LAB & $1 / 7(14 \%)$ & $2 / 8(38 \%)$ & $5 / 10(50 \%)$ \\
Enterobacteria & $1 / 7(1 \%)$ & $0 / 8(0 \%)$ & $1 / 10(10 \%)$ \\
Ileal mucosa-associated & & & \\
$\quad$ bacteria $\log _{10} / \mathrm{g}$ tissue & & & \\
Aerobes & $5.4(0.9)$ & $4.9(1.5)$ & $5.4(0.5)$ \\
Anaerobes & $5.7(0.7)$ & $5.3(1.4)$ & $5.6(0.8)$ \\
LAB & $5.4(0.3)$ & $4.9(1.3)$ & $4.6(2.1)$ \\
Enterobacteria & $2.4(2.4)$ & $4.5(1.4) \|$ & $5.0(1.4) \dagger$ \\
\hline
\end{tabular}

* Different vs. CTL $(p<0.05)$.

$\dagger$ Different $v s$. mother-reared rats $(p<0.01)$.

$\ddagger$ Results are shown as number of rats with positive spleen culture/total number of rats; percentages are parenthesized.

$\S \mathrm{LAB}$, lactic acid producing bacteria.

II Results are shown as medians; interquartiles (Q1-Q3) are parenthesized.

$\|$ Different $v s$. mother-reared rats $(p<0.05)$.

claudin-2 and claudin-3, relative to GAPDH expression, were not altered by dietary GOS/inulin (claudin-2: 0.86 (1.11) vs. 0.73 (0.26); claudin-3: 0.89 (0.54) vs. 0.68 (1.20) for CTL and GOS/in, respectively). The expression of ZO-1, however, was $40 \%$ lower in the GOS/inulin compared with CTL [0.59 (0.11) vs. 0.93(0.67), respectively; $p=0.02$ ].

Ileal mucosa-associated bacteria. Numbers of ileal mucosaassociated aerobes, anaerobes, lactic acid producing bacteria, and enterobacteria in the ileum were not different between GOS/inulin and CTL (Table 3). However, the number of ileal mucosa-associated enterobacteria in mother-reared rats was significantly lower, compared with both CTL and GOS/inulin (Table 3).

\section{DISCUSSION}

The current study demonstrates that in neonatal rat receiving enteral nutrition, supplementation with a mixture of GOS/ inulin 1) enhances fecal excretion, and luminal concentrations of L-lactate and acetate in the large intestine, 2) exerts a trophic effect on cecal mucosa, and 3) increases BT toward spleen. To the best of our knowledge, the current study is the first to demonstrate such an impact which has potentially important implications as the potential risks and benefits of increased $\mathrm{BT}$ remain to be explored.

In view of the literature, increased BT following supplementation with prebiotics in the neonatal period was not unexpected. As a matter of fact, an increased translocation of Salmonella toward spleen was reported in adult rats fed a low-calcium diet enriched with fructooligosaccharides or inulin when animals were infected with this pathogen $(13,19)$. The authors hypothesized that the rapid fermentation of the prebiotics led to high ceco-colonic concentrations of organic acids that impaired the intestinal barrier function. Consistent with the latter hypothesis, the increased BT observed in the current study was concomitant with a rise in the concentration of organic acids in the ceco-colonic luminal content in rats fed GOS/inulin. Furthermore, regardless of regimen, the concentration of total organic acids was significantly higher in rats displaying BT, compared with rats without BT [87.2 (26.2) vs. $69.8(26.5) \mu \mathrm{mol} / \mathrm{g}$, respectively; $p<0.01]$.

Whether these concentrations of organic acids can impair the barrier function is debatable since Lin et al. showed that in situ perfusions of $300 \mathrm{mM}$ of SCFA, but not $150 \mathrm{mM}$, induced colonic mucosal damage in newborn rats (10). In the current study, the mean concentration of organic acids in pooled ceco-colonic content at d18 never exceeded $150 \mu \mathrm{mol} / \mathrm{g}$ but 1) the concentration of organic acids was probably higher in the cecum (i.e., the major fermentation site for oligosaccharides in rodent) than in the colon, and 2) luminal SCFA concentrations only partially reflect SCFA production because of absorption. So it cannot be excluded that higher concentrations-close to those able to produce mucosal lesion-were reached at some point in time in the cecum or colon of our supplemented pups.

However, other findings argue against the hypothesis of a "toxic" mucosal injury mediated by organic acids. First, whereas the enhanced BT associated with fructooligosaccharides and inulin supplementation in adult rats was dependent on calcium deficiency (19), our rats were fed a normalcalcium diet. Moreover, in the current study and conversely to Ten Bruggencate and coworkers $(13,14)$, GOS/inulin supplementation altered neither the histologic characteristics of cecum and colon (data not shown) nor the permeability of cecum and colon to $4 \mathrm{KDa}$ dextran. Although colonic permeability was measured across unstripped intestinal walls, it is unlikely that the underlying muscle layers have masked differences in the permeability across the epithelium per se since the flux of 
$4 \mathrm{KDa}$ probes is not altered by these layers in distal colon of adult rabbit (20). We also failed to detect any alteration in the colonic expression of genes encoding for claudin-2 and claudin-3, two proteins that are constitutive of tight junctions, and presumably form the pores that regulate paracellular pathway (21). Conversely, we detected a small significant downregulation of ZO-1 mRNA expression, but earlier studies suggest that deletion of ZO-1 is not associated to altered paracellular permeability (22). Taken together and subject to confirmation by in vivo measurement of permeability, these results suggest that the increased BT we observed was not induced by alterations in the ceco-colonic permeability.

The increased BT detected in the GOS/inulin group may have originated from the small intestine, rather than colon. Two lines of evidence, possibly interconnected, support such a hypothesis. First, animal studies suggest that BT in newborn is largely related to the colonization of small intestine rather than colon (23). Whether excessive small intestinal proliferation of luminal microbiota occurred in our study following GOS/inulin supplementation could not be verified due to the insufficient mass of intestinal contents. However, we did not observe any difference in ileal mucosa associated microbiota between the two regimens, which argues against this hypothesis. Second, the ileal associated lymphoid tissue, rich in $\mathrm{M}$ cells that are known pathways for BT, is potentially responsive to prebiotics. Interaction between prebiotics and immune cells relies on several mechanisms including local or systemic indirect effects mediated by enhanced production of immunoregulatory SCFA or a shift in the composition of the intestinal microbiota, or direct effect mediated via carbohydrate receptors (24). In the current study, an indirect effect through enhanced production of SCFA or changes in the microbiotal composition is unlikely in the small intestine since transit in this segment, which is very fast in newborn rats (25), is hardly compatible with significant fermentation. Moreover, approximately $90 \%$ of an ingested dose of fructooligosaccharides and inulin have been shown to reach the colon of humans and/or animals (26). It is therefore tempting to speculate that modulation of the ileal-associated lymphoid tissue by GOS/inulin might account for the increased BT in the current study. Putative mechanisms include either a direct local effect via carbohydrate receptors, or a systemic one, through fermentation of prebiotics in the large intestine.

Regardless of the mechanism underlying this increased BT, it raises the question of the safety of the GOS/inulin for newborn infants. The consequences of BT on the host health are not fully understood. BT has long been considered detrimental, particularly in preterm infants, as it has been directly involved in the pathogenesis of necrotizing enterocolitis, along with several other factors (27). Yet, until now, supplementation of infant formulas with GOS/inulin has not been associated with adverse effects in full-term nor in preterm infants (28). If an increased BT associated to the supplementation of GOS/inulin were to be confirmed in infants, this effect might not necessarily be harmful. Indeed, in neonatal animals (29), as well as in human infants (30), a high incidence of BT has been reported to occur naturally, without any obvious adverse consequences. Since the occurrence of BT paralleled the development of the gut-associated lymphoid tissue, the authors speculated that BT could be involved in the postnatal maturation of the immune system.

In conclusion, using an animal model of artificial feeding we demonstrate that feeding with a GOS/inulin-enriched formula in the neonatal period enhances the metabolic activity of colonic microbiota, as observed in human infants (2), and increases BT toward spleen at $\mathrm{d} 18$ as well. This effect did not persist at $\mathrm{d} 40$, after $20 \mathrm{~d}$ of weaning with a normal, prebioticfree, solid diet. The mechanisms underlying this increased BT are unclear, and do not reflect any obvious alteration in colonic barrier function. Further investigation of the effect of GOS/inulin on the small intestinal immune system is warranted to establish whether this stimulation of BT is beneficial to the maturation of the immune system.

\section{REFERENCES}

1. Fanaro S, Boehm G, Garssen J, Knol J, Mosca F, Stahl B, Vigi V 2005 Galactooligosaccharides and long-chain fructo-oligosaccharides as prebiotics in infant formulas: a review. Acta Paediatr Suppl 94:22-26

2. Knol J, Scholtens P, Kafka C, Steenbakkers J, Gro S, Helm K, Klarczyk M, Schopfer H, Bockler HM, Wells J 2005 Colon microflora in infants fed formula with galactoand fructo-oligosaccharides: more like breast-fed infants. J Pediatr Gastroenterol Nutr 40:36-42

3. Arslanoglu S, Moro GE, Boehm G 2007 Early supplementation of prebiotic oligosaccharides protects formula-fed infants against infections during the first 6 months of life. J Nutr 137:2420-2424

4. Moro G, Arslanoglu S, Stahl B, Jelinek J, Wahn U, Boehm G 2006 A mixture of prebiotic oligosaccharides reduces the incidence of atopic dermatitis during the first six months of age. Arch Dis Child 91:814-819

5. Anand RJ, Leaphart CL, Mollen KP, Hackam DJ 2007 The role of the intestinal barrier in the pathogenesis of necrotizing enterocolitis. Shock 27:124-133

6. Colome G, Sierra C, Blasco J, Garcia MV, Valverde E, Sanchez E 2007 Intestinal permeability in different feedings in infancy. Acta Paediatr 96:69-72

7. Hamer HM, Jonkers D, Venema K, Vanhoutvin S, Troost FJ, Brummer RJ 2008 Review article: the role of butyrate on colonic function. Aliment Pharmacol Ther 27:104-119

8. Kanauchi O, Iwanaga T, Mitsuyama K, Saiki T, Tsuruta O, Noguchi K, Toyonaga A 1999 Butyrate from bacterial fermentation of germinated barley foodstuff preserves intestinal barrier function in experimental colitis in the rat model. J Gastroenterol Hepatol 14:880-888

9. Popoff MR, Jolivet-Reynaud C, Carlier JP 1987 Cytotoxic activity of Clostridium butyricum supernatants induced by butyrate. FEMS Microbiol Lett 43:95-100

10. Lin J, Nafday SM, Chauvin SN, Magid MS, Pabbatireddy S, Holzman IR, Babyatsky MW 2002 Variable effects of short chain fatty acids and lactic acid in inducing intestinal mucosal injury in newborn rats. J Pediatr Gastroenterol Nutr 35:545-550

11. Nafday SM, Chen W, Peng L, Babyatsky MW, Holzman IR, Lin J 2005 Short-chain fatty acids induce colonic mucosal injury in rats with various postnatal ages. Pediatr Res 57:201-204

12. Edwards CA, Parrett AM 2002 Intestinal flora during the first months of life: new perspectives. Br J Nutr 88:S11-S18

13. Bovee-Oudenhoven IM, Ten Bruggencate SJ, Lettink-Wissink ML, van der Meer R 2003 Dietary fructo-oligosaccharides and lactulose inhibit intestinal colonisation but stimulate translocation of salmonella in rats. Gut 52:1572-1578

14. Ten Bruggencate SJ, Bovee-Oudenhoven IM, Lettink-Wissink ML, Van der Meer R 2005 Dietary fructooligosaccharides increase intestinal permeability in rats. J Nutr 135:837-842

15. Hall WG 1975 Weaning and growth of artificially reared rats. Science 190:13131315

16. Kanno T, Koyanagi N, Katoku Y, Yonekubo A, Yajima T, Kuwata T, Kitagawa H, Harada E 1997 Simplified preparation of a refined milk formula comparable to rat's milk: influence of the formula on development of the gut and brain in artificially reared rat pups. J Pediatr Gastroenterol Nutr 24:242-252

17. Jouany JP, Senaud J 1982 [Effect of rumen ciliates on the digestion of different carbohydrates in sheep. I.-Utilization of cell wall carbohydrates (cellulose and hemicellulose) and of starch]. Reprod Nutr Dev 22:735-752

18. Moolenbeek C, Ruitenberg EJ 1981 The "Swiss roll": a simple technique for histological studies of the rodent intestine. Lab Anim 15:57-59

19. Ten Bruggencate SJ, Bovee-Oudenhoven IM, Lettink-Wissink ML, Katan MB, Van Der Meer R 2004 Dietary fructo-oligosaccharides and inulin decrease resistance of rats to salmonella: protective role of calcium. Gut 53:530-535

20. Ghandehari H, Smith PL, Ellens H, Yeh PY, Kopecek J 1997 Size-dependent permeability of hydrophilic probes across rabbit colonic epithelium. J Pharmacol Exp Ther 280:747-753 
21. Van Itallie CM, Anderson JM 2004 The molecular physiology of tight junction pores. Physiology (Bethesda) 19:331-338

22. Umeda K, Matsui T, Nakayama M, Furuse K, Sasaki H, Furuse M, Tsukita S 2004 Establishment and characterization of cultured epithelial cells lacking expression of ZO-1. J Biol Chem 279:44785-44794

23. Urao M, Moy J, Van Camp J, Drongowski R, Altabba M, Coran AG 1995 Determinant of bacterial translocation in the newborn: small bowel versus large bowel colonization. J Pediatr Surg 30:831-836

24. Seifert S, Watzl B 2007 Inulin and oligofructose: review of experimental data on immune modulation. J Nutr 137:2563S-2567S

25. Tomomasa T, Itoh K, Hyman PE, Kuroume T 1991 Oral neurotensin increases gastrointestinal transit in suckling rats. J Pediatr Gastroenterol Nutr 13:77-82
26. Cummings JH, Macfarlane GT, Englyst HN 2001 Prebiotic digestion and fermentation. Am J Clin Nutr 73:415S-420S

27. Deitch EA 1994 Role of bacterial translocation in necrotizing enterocolitis. Acta Paediatr Suppl 396:33-36

28. Agostoni C, Axelsson I, Goulet O, Koletzko B, Michaelsen KF, Puntis JW, Rigo J, Shamir R, Szajewska H, Turck D 2004 Prebiotic oligosaccharides in dietetic products for infants: a commentary by the ESPGHAN Committee on Nutrition. J Pediatr Gastroenterol Nutr 39:465-473

29. Urao M, Teitelbaum DH, Drongowski RA, Coran AG 1996 The association of gut-associated lymphoid tissue and bacterial translocation in the newborn rabbit J Pediatr Surg 31:1482-1487

30. Gebbers JO, Laissue JA 2004 Bacterial translocation in the normal human appendix parallels the development of the local immune system. Ann N Y Acad Sci 1029:337-343 